\title{
3 Land und Verräumlichungspraktiken
}

Um den Zusammenhang von Land und Praktiken der (Neu-)Verräumlichung zu untersuchen, beziehen wir uns auf den im SFB 1199 entwickelten Ansatz, der zwischen vielfältigen Verräumlichungen als Ergebnis jeglicher Art von sozialer Interaktion und Raumformaten als Formen verstetigter, institutionalisierter, performativ artikulierter und intersubjektiv reflektierter Räume unterscheidet. ${ }^{1}$ Verräumlichungen und Raumformate entstehen dabei in enger Verflechtung mit Imaginationen, die ebendiese Raumformate oder aber deren Herausforderung und Modifikation enthalten können.

Der Begriff der Imaginationen ist hilfreich, um hervorzuheben, dass wirtschaftliche, finanzkapitalistische, politische oder kulturelle Interessen, die Akteur:innen innerhalb von Landprojekten verfolgen, nicht unmittelbar als das Ergebnis zunehmender Kommodifizierung und Finanzialisierung oder Mobilisierung und Widerstand zu sehen sind. ${ }^{2}$ Alle diese Prozesse beinhalten vielmehr spezifische Vorstellungen von Land und damit verbundene Annahmen, Visionen, Ideen, Hoffnungen oder Träume im Hinblick darauf, was Land ist, sein kann oder sein soll. Diese Landimaginationen können hegemonial, unterdrückt oder marginalisiert werden. Sie können sowohl „normalisiert“ als auch Kern für Anstoß und Widerstand sein und damit zum Mittel der Unterdrückung oder zur Triebfeder der Befreiung werden. Landimaginationen interagieren somit auf vielfältige Weise mit Praktiken der Verräumlichung und ihren Verstetigungen zu Raumformaten. In der Land Rush-Debatte wurden Landimaginationen lediglich implizit behandelt. Wir rücken sie ins Zentrum und fragen: Welche Landimaginationen gehen mit aktuellen Landtransformationen einher, wie entfalten sie sich und was hat dies für Implikationen?

Analytisch unterscheiden wir dabei zwei Dimensionen innerhalb von Imaginationen. Sie können Raumzusammenhänge erstens eher implizit abbilden und Verräumlichungspraktiken damit unterschwellig, unterbewusst oder unreflektiert beeinflussen. Auf diese Dimension zielt insbesondere der Begriff der environmental imaginaries in der Umweltgeschichte und Politischen Ökologie. Im Zentrum steht die Frage, wie Gesellschaften Natur und Umwelt kollektiv konstruieren,

1 Ergänzend dazu werden im Rahmen des SFB 1199 Raumordnungen untersucht, die sich im Ergebnis von Aushandlungsprozessen aus der Kombination verschiedener Raumformate ergeben. Raumordnungen werden in diesem Beitrag nicht betrachtet.

2 Sippel und Visser, „Reimagining Land“. 
interpretieren und über diese kommunizieren. ${ }^{3}$ Zweitens können Imaginationen Landprojekte explizit und bewusst vorantreiben, wenn Akteur:innen gezielt bestimmte (Neu-)Verräumlichungen von Land anstreben. In dieser zweiten Dimension wird Imaginieren als eine soziale Praxis verstanden, innerhalb derer sich Menschen andere oder neue Welten ausmalen und diese dann aktiv umzusetzen suchen. ${ }^{4}$ Imaginieren, so schreibt beispielweise der britische Sozialanthropologe Tim Ingold, ist mehr als die reine Fähigkeit, mentale Bilder oder Repräsentationen $\mathrm{zu}$ konstruieren, es meint auch „to participate from within, through perception and action, in the very becoming of things“. ${ }^{5}$ Diese aktive Komponente des Imaginierens ist auch in Jasanoffs Konzept der sociotechnical imaginaries angesprochen, die sie als „collectively held, institutionally stabilized, and publicly performed visions of desirable futures“ " definiert. ${ }^{6}$

Innerhalb dieser zweiten Dimension lassen sich zwei Arten des aktiven, expliziten Imaginierens unterscheiden. So können zum einen bereits existierende Landimaginationen auf neue Räume, Sphären oder Zeitperioden übertragen werden (beispielweise die Ausbreitung der Konzeption von Land als Eigentum und Ware im Zuge der europäischen Kolonisation). Zum anderen können Landimaginationen als „Gegenimaginationen“ entworfen werden, um bestehende oder dominante Imaginationen herauszufordern und zu ersetzen. Panikkar und Tollefson beschreiben eine solche alternative Form der Landimagination am Beispiel der Anti-Kieselminen-Koalition in Alaska. ${ }^{7}$ Diese hat unter anderem „Subsis-

3 R. Peet und M. Watts, Liberation Ecologies. Environment, Development, Social Movements, London: Routledge, 1996; J. T. Nesbitt und D. Weiner, „Conflicting Environmental Imaginaries and the Politics of Nature in Central Appalachia“, Geoforum 32 (2001) 3, S. 333-349; A. McGregor, „Sustainable Development and ,Warm Fuzzy Feelings'. Discourse and Nature within Australian Environmental Imaginaries“, Geoforum 35 (2004) 5, S. 593-606; D. K. Davis, „Imperialism, Orientalism, and the Environment in the Middle East“", in: D. K. Davis, E. Burke (Hrsg.) Environmental Imaginaries of the Middle East and North Africa, Athens: Ohio University Press, 2011, S. 1-22; T. Mitchell, „Are Environmental Imaginaries Culturally Constructed?“, in: ebd., S. 265-273.

4 S. Daniels, „Geographical Imagination“, Transactions of the Institute of British Geographers 36 (2011) 2, S. 182-187; J. Watkins, „Spatial Imaginaries Research in Geography. Synergies, Tensions, and New Directions“, Geography Compass 9 (2015) 9, S. 508-522.

5 T. Ingold, „Introduction“, in: M. Janowski und T. Ingold (Hrsg,) Imagining Landscapes: Past, Present, and Future, Farnham: Ashgate, 2015, S. 1-18, hier S. 3.

6 S. Jasanoff, „Imagined and Invented Worlds“, in: S. Jasanoff und S.-H. Kim (Hrsg.), Dreamscapes of Modernity. Sociotechnical Imaginaries and the Fabrication of Power, Chicago: University of Chicago Press, 2015, S. 321-341, hier S. 322.

7 B. Panikkar und J. Tollefson, „Land as Material, Knowledge and Relationships: Resource Extraction and Subsistence Imaginaries in Bristol Bay, Alaska“, Social Studies of Science 48 (2018) 5, S. $715-739$. 
tenzimaginationen“ mobilisiert, um dominanten Imaginationen des Ressourcenextraktivismus entgegenzutreten.

Ergänzend zu diesen unterschiedlichen Dimensionen von Landimaginationen möchten wir den Zusammenhang zwischen Land und konkreten Praktiken der Verräumlichung sowie ihrem Gerinnen zu Raumformaten in den Blick nehmen. Im Zuge des spatial turn hat sich in den Sozialwissenschaften das Verständnis von Raum grundlegend gewandelt. Raum wurde nicht mehr als ,gegeben“ betrachtet, sondern als menschengemacht. Verräumlichung wurde damit zentrale Dimension und Resultat menschlichen Handelns zugleich. Der Fokus auf den sozialen Raum hat allerdings dessen physische Komponenten zeitweise in den Hintergrund treten lassen - teils konnte der Eindruck entstehen, Raum sei allein „sozial“ verfasst und entbehre jeglicher materiellen Qualität. Im Zuge des New Materialism haben Bezüge auf die physische und gebaute Welt und ihre komplexe Interaktion oder Ko-Produktion mit dem Sozialen eine neue Bedeutung erfahren. Diese schlagen sich, so Rao, auch in einer Erweiterung der Raumtheorie in den Sozialwissenschaften nieder. Die physische Einbettung räumlichen Handelns werde konsequenter in einem komplexen Konstruktionsbegriff erfasst, der die dialektische Beziehung von Raum als sowohl sozio-materiell vorgegeben als auch gemacht zu denken vermag. ${ }^{8}$ So wird beispielsweise nach den Rückkopplungseffekten zwischen den Aktivitäten intentionaler und nicht-intentionaler Akteur:innen, der raumgestaltenden Wirkung der Neujustierung der Beziehungen zwischen Menschen, Tieren, Mikroben oder Landschaften, oder dem Aufforderungscharakter physischer Räume gefragt.

Die Verschmelzung sozio-kultureller, polit-ökonomischer und physisch-materieller Dimensionen von Land unterstreicht diese Erweiterung des Raumbegriffs. Wir haben eingangs bereits auf die vielschichtigen Dimensionen von Land verwiesen - als unabdingbar für das menschliche Überleben, Symbol von Identität und Zugehörigkeit ebenso wie Eroberung und Vertreibung. Prozesse der Territorialisierung - Imperialismus, Kolonialismus, Nationenbildung - sind aufs Engste mit Land verflochten und dies sehr viel stärker als - so lässt sich argumentieren mit anderen Naturelementen. Sogar Wasser - ebenfalls von großer sozio-kultureller Bedeutung und unabdingbar für das Überleben von Menschen - ist weniger ein Symbol für Territorialität und Identität. Land hat zugleich eine Reihe physisch-materieller Eigenschaften, die für die Möglichkeiten seiner Verräumlichung und seine Integration in Verräumlichungsprozesse zentral sind. Eine Eigenschaft

8 U. Rao, Praktiken der Verräumlichung (Dialektik des Globalen, Kernbegriffe, 8), Berlin: De Gruyter Oldenbourg, 2021 (im Erscheinen). Siehe auch S. Low, Spatializing Culture. The Ethnography of Space and Place, New York: Routledge, 2017. 
von Land ist sein verhältnismäßig „fester“ und beständiger Zustand. ${ }^{9}$ Tiere bewegen sich. Pflanzen wachsen, dehnen sich aus und werden über weite Strecken hinweg als Holz und Biomasse bewegt. Auch unterirdische Ressourcen wie Öl oder Mineralien werden über den Globus transportiert. Die Materialität von Land verhindert diese Art des Transports weitgehend, was seine Mobilität - zumindest über die Lebensspanne eines Menschen hinweg - stark einschränkt. Land bleibt auch dann noch übrig, wenn eine Schicht des Landes (oder Erdbodens) abgetragen wird..$^{10}$ Land fungiert ebenfalls häufig als „Geburtsort“ anderer Ressourcen. Es bringt als Agrarland oder Wald vielfältige Ackerfrüchte hervor, kann zahlreiche Mineralien enthalten und hat jüngst durch seine Fähigkeit (in Kombination mit Pflanzen), Kohlenstoffdioxid aus der Atmosphäre zu speichern, an Wert gewonnen. Land ist darüber hinaus - im Gegensatz zu anderen subterranen Ressourcen - eine erneuerbare Ressource in seinen Funktionen als Agrarland, Waldland, Biogasland oder Land für Solaranlagen und Windräder (sofern es nachhaltig genutzt wird). Die Materialität von Land spendet Leben, erlaubt das Anbauen, Sammeln und Jagen von Nahrung und ermöglicht den Bau einer Behausung zum Wohnen. Kurzum, menschliches Leben ist undenkbar ohne Land, insbesondere in seiner physio-materiellen Dimension. Nicht zuletzt vor dem Hintergrund ebendieser vielfältigen physio-materiellen Eigenschaften wurde Land in den meisten menschlichen Zusammenhängen über Raum und Zeit hinweg ein besonders hoher Wert zugesprochen.

Wie nun wird Land im Zuge aktueller Landtransformationen (re-)imaginiert und (neu) verräumlicht? Vier mit einander verflochtene Dimensionen der (Re-)

9 Wenngleich sehr viel beständiger und „fester“ im Vergleich zu anderen Naturelementen, so lässt sich auch die Festigkeit oder Beständigkeit von Land diskutieren. Es existieren durchaus Landimaginationen, die einen eher fluiden Charakter von Land betonen, beispielweise imaginieren Bauern in der stark von Erosion betroffenen mexikanischen Bergregion Patzcuaro Basin Land als beweglich (Barrera-Bassols und Zinck, ,Land Moves and Behaves'). Auch Sud hat jüngst die Nicht-Festigkeit (unfixity) von Land in der Politik des indischen Staates beschrieben (N. Sud, „The Unfixed State of Unfixed Land“, Development and Change 51 [2019] 5, S. 1-24). Ebenso können die Grenzen zwischen Land und Wasser durchaus fluide sein (T. Richardson, „Where the Water Sheds. Disputed Deposits at the Ends of the Danube“, in: M. Bozovic und M. D. Miller [Hrsg.], Watersheds. The Poetics and the Politics of the Danube River, Boston: Academic Studies Press, 2016, S. 307-336; T. Richardson, „The Terrestrialization of Amphibious Life in a Danube Delta ,Town on Water“", Suomen Antropologi: Journal of the Finish Anthropological Society 43 [2018] 2, S. 3-29; L. Cortesi, „The Muddy Semiotics of Mud“, Journal of Political Ecology 25 [2018], S. 617637).

10 Außer in Fällen, in denen solche Mengen von Land abgetragen werden, dass Gewässer entstehen, zum Beispiel durch Tagebau. 
Imagination und (Neu-)Verräumlichung von Land lassen sich im Rahmen aktueller Landprojekte identifizieren:

1. die Neu-Formatierung von Land im Zuge der Schaffung neuer Anlagegeographien,

2. die besonderen Herausforderungen, die die Materialität von Land an seine Verräumlichung stellt,

3. die De- und Re-Regulierung von Land vor dem Hintergrund der Zunahme ausländischer Landkäufe und

4. lokale Aushandlungen und transregionale Mobilisierungen im Kontext von Land. 